\title{
Effects of iron supplementation of low-birth-weight infants on cognition and behavior at 7 years: a randomized controlled trial
}

\author{
Staffan K. Berglund ${ }^{1}$, Anna Chmielewska ${ }^{1}$, Josefine Starnberg ${ }^{1}$, Björn Westrup ${ }^{2}$, Bruno Hägglöf ${ }^{3}$, Mikael Norman ${ }^{4}$ and \\ Magnus Domellöf ${ }^{1}$
}

BACKGROUND: Low-birth-weight infants (LBW) are at an increased risk of iron deficiency that has been associated with impaired neurodevelopment. We hypothesized that iron supplementation of LBW infants improves cognitive scores and reduces behavioral problems until school age.

METHODS: We randomized 285 marginally LBW (2,000$2,500 \mathrm{~g}$ ) infants to receive 0,1 , or $2 \mathrm{mg} / \mathrm{kg} /$ day of iron supplements from 6 weeks to 6 months of age. At 7 years of age, 205 participants were assessed regarding cognition using Wechsler Intelligence Scale for Children (WISC-IV) and behavior using the parental questionnaires Child Behavior Checklist (CBCL) and Five to Fifteen (FTF).

RESULTS: There were no significant differences between the intervention groups in WISC-IV or FTF. However, the CBCL scores for externalizing problems were significantly different, in favor of supplemented children $(P=0.045)$. When combining the supplemented groups, they had significantly lower scores for externalizing behavior compared with placebo (median (interquartile range): $44[34 ; 51]$ vs. 48.5 [41;56] $P=0.013)$, and their risk ratio (95\% confidence interval) for a total behavioral score above the cutoff for clinical problems was $0.31(0.09-1.0), P=0.054$.

CONCLUSION: Lower scores of externalizing behavior in supplemented children support our previous findings at 3 years, and suggest that iron supplementation may have longlasting effects on behavioral functions.

B eing born with low birth weight (LBW, $<2,500 \mathrm{~g}$ ) is associated with increased risk of impaired neurodevelopment $(1,2)$. This association has been clearly demonstrated in very LBW infants $(<1,500 \mathrm{~g})(1,3,4)$, but lower scores of cognitive and behavioral performance have also been observed in those born with moderately LBW (1,5002,500 g) (3-9).

It is well known that LBW infants are at risk of iron deficiency (ID) due to their high iron needs in relation to the iron endowment at birth $(10,11)$. As ID has been associated with poor neurodevelopment in normal-birth-weight infants
(12), it is reasonable to assume that ID may contribute to the impaired neurodevelopment observed in LBW infants. This is also the main reason why iron supplementation is generally recommended to preterm and LBW infants $(11,13)$. However, long-term neurodevelopmental effects of iron supplementation in LBW populations have not previously been explored in randomized trials, and as both benefits and possible adverse events have been reported from randomized trials in term infants, further knowledge regarding both short- and longterm effects of iron supplementation to LBW infants is warranted (10,14-18). Moreover, there has been a particular lack of knowledge regarding the effects of iron supplementation to the largest subgroup of LBW children, those born with marginally LBW $(2,000-2,500 \mathrm{~g})$. This heterogenous group of LBW infants includes both late preterm and term infants, as well as infants born small for gestational age (SGA), and represents as much as $3-5 \%$ of all infants in high-income countries $(19,20)$ and $>15 \%$ in some low-income countries (21).

We have recently shown in a randomized controlled trial that iron supplementation between 6 weeks and 6 months of life given to Swedish marginally LBW infants decreased the risk of ID and ID anemia at 6 months (18), and the risk of ID at 12 months (22). We also observed a decreased risk of behavioral problems, but no effect on cognitive scores at 3.5 years of age (23). In the present study, we hypothesized that the early iron intervention would improve neurobehavioral function at school age, and we assessed cognitive scores using WISC and behavior using two validated parental questionnaires at 7 years of age.

\section{METHODS}

\section{Study Design and Participants}

This double-blinded randomized controlled trial was conducted at Umeå University Hospital (Umeå, Sweden) and Karolinska University Hospital (Stockholm, Sweden) between March 2004 and November 2007. Infants $(n=285)$ were included at 6 weeks of age. The inclusion criteria were birth weight of 2,000-2,500 g, no chronic diseases diagnosed at inclusion, and no previous blood transfusion or iron supplementation. Exclusion criteria in the present analyses were anemia (hemoglobin $<90 \mathrm{~g} / \mathrm{l})$ at baseline $(n=16)$, other

\footnotetext{
${ }^{1}$ Department of Clinical Sciences, Pediatrics, Umeå University, Umeå, Sweden; ${ }^{2}$ Division of Neonatology, Department of Women and Child Health, Karolinska Institutet, Stockholm, Sweden; ${ }^{3}$ Department of Clinical Sciences, Child and Adolescent Psychiatry, Umeå University, Umeå, Sweden; ${ }^{4}$ Division of Pediatrics, Department of Clinical Science, Intervention and Technology, Karolinska Institutet, Stockholm. Correspondence: Staffan K. Berglund (staffan.berglund@umu.se)

Received 28 March 2017; accepted 16 July 2017; advance online publication 25 October 2017. doi:10.1038/pr.2017.235
} 


\section{Articles | Berglund et al.}

hematological disorders diagnosed during the study $(n=2)$, or a diagnosed genetic disorder that includes neurological impairment ( $n=3$; Williams syndrome, 22q11 syndrome, and Duchenne muscular dystrophy). We used delivery records to identify eligible infants meeting the inclusion criteria and approached the parents at the delivery unit, at the neonatal clinic, or at home (18). Another 95 children were included as a reference group before a previously described follow-up at 3.5 years of age (23). The reference group was matched by sex, birth hospital, and the time of birth to every third LBW participant. Additional inclusion criteria for the reference group were gestational age of 37-42 weeks, birth weight of 2,501$4,499 \mathrm{~g}$, not admitted to a neonatal unit, and no diagnosed disease until 6 weeks of age. Children and parents accepting participation gave written informed consent, and the study was approved by the Ethical Review Boards in Umeå and the Stockholm and was registered with ClinicalTrials.gov, number NCT00558454.

\section{Intervention}

The intervention procedure has been described in detail previously (18). Stratified by sex and study center, all LBW participants were randomized, by means of a computer-generated randomization list, to receive 0 (placebo), 1 , or $2 \mathrm{mg} / \mathrm{kg} /$ day of iron supplements (ferrous succinate drops) from 6 weeks to 6 months of age. Parents and all staff involved in data collection were blinded to the treatment assignment during the intervention and throughout the 7-year follow-up. The supplements (or placebo) were delivered in identical bottles, with a similar taste and color and divided into two daily doses. The individual dose of iron supplements/placebo was adjusted according to the infant's weight at inclusion and at 12 and 19 weeks. Compliance to the intervention was monitored using a daily checklist. An intake of less than $70 \%$ was considered as poor compliance with the intervention and this was registered in 64 (24\%) of the participants. We have previously shown that there were no significant differences between the groups in the prevalence of poor compliance, or in dropout rate during the intervention (18).

Apart from the intervention, parents were encouraged to follow the general Swedish infant dietary recommendations, including recommended exclusive breastfeeding until 4-6 months, and ironfortified formula (typically containing $4-8 \mathrm{mg} / \mathrm{l}$ ) in cases of nonexclusive breastfeeding.

\section{Data Collection During Intervention}

At inclusion, perinatal and background characteristics were collected using delivery records and a parental questionnaire. Baseline data included weight, length, and head circumference at birth, Apgar score, gestational age, and neonatal diagnoses as well as information regarding the parents' country of birth, age, and smoking habits. During the intervention, participating infants visited the study center at 6 weeks, 12 weeks, 19 weeks, and 6 months. The visits included anthropometric assessment (weight, length, and head circumference) and, before each visit, the parents were instructed to complete a 3day food diary analyzed for the mean daily iron intake (18).

At 6 weeks, 12 weeks, and 6 months of age, a venous blood sample was drawn by an experienced research nurse. Blood was analyzed for iron status (hemoglobin, ferritin, mean corpuscular volume, iron, transferrin, and transferrin saturation) and the prevalences of ID and ID anemia were assessed, as described elsewhere $(18,22)$. If anemia was confirmed by two separate measures at 12 weeks (hemoglobin $<95 \mathrm{~g} / \mathrm{l})$, the infant was referred to a pediatrician for further evaluation and treatment. Following this procedure, nine children were prescribed to unblinded iron supplements due to suspected ID anemia. These participants remained in the study according to an intention to the treat principle.

\section{Data Collection at Follow-up}

All included LBW children and the 95 controls were invited to the present follow-up at 7 years of age. Before the visit, parents were asked to answer a questionnaire including questions on parental education, and family structure, adding an update on the sociodemographic characteristics. At the visit, blood was drawn and sent to the hospital laboratory connected to each of the two study sites. Analysis was performed for hemoglobin and serum levels of ferritin, transferrin, iron, and transferrin saturation.

During the visit at 7 years of age, a psychometric intelligence quotient (IQ) was assessed using the validated Wechsler Intelligence Scale for Children-fourth edition (WISC-IV), performed by an experienced authorized pediatric psychologist. The WISC-IV test includes four IQ subscales (verbal comprehension, perceptual reasoning, working memory, and processing speed), as well as a combined full-scale IQ. All five scales are standardized with a mean of 100 and a SD of 15 ( (ref. 24)). In the present analyses, cognitive impairment was defined as an IQ below 85 .

During or before the visit at 7 years of age, the parents were asked to complete the two parental questionnaires Child Behavioral Checklist for ages 6-18 (CBCL) (25) and Five to Fifteen (FTF), assessing various types of behavioral and emotional problems (26). CBCL is a widely used, standardized measure to be completed by a parent/caretaker (27). It provides ratings for 20 competence and 120 problem items, and parents rate how true each item is now, or was within the past 6 months, using a three-point scale $(0=$ not true, $1=$ somewhat true, and $2=$ very true or often true). The CBCL for ages 6-18 yields scores on internalizing, externalizing, and total behavioral problems. The scores are reported as T-scores (mean 50 and SD 10) and the cutoff for clinical and subclinical problems is set to $>63$ ( $>90$ th percentile) and $>59$ ( $>83$ rd percentile), respectively, based on a US pediatric population. The CBCL for ages 6-18 can be further subdivided into nine nonstandardized subscales (syndrome scales). We converted these subscale scores into SD scores (SDS) using the results from our reference group.

The FTF is a parental questionnaire that includes 181 statements assessing the symptoms of behavioral or developmental problems. The parents rate the statements as either $0=$ does not apply, $1=$ applies sometimes or to some extent, and $2=$ definitely applies. The items are arranged into eight different domains (memory, learning, language, executive functions, motor skills, perception, social skills, and emotional/behavioral problems), each with a mean score ranging from 0 to 2 ( (ref. 26)). In the present study, we converted the mean domain scores of the parent FTF to SDS in relation to the scores for the Swedish age- and gender-specific reference population (26), and defined impairment of a domain if SDS was above 2.0.

\section{Statistical Analysis}

In the power calculation for the present study, we assumed a dropout rate of $20 \%$ and a poor compliance rate of $15 \%$, and aimed, with a power of $80 \%$ and a significance level of 0.05 , to detect an effect size between two groups of $0.5 \mathrm{SD}$, corresponding to 7.5 points in total IQ. In the present analyses, we used IBM SPSS Statistics for Windows, Version 22.0 (IBM Corp., Armonk, NY, USA) to perform two-tailed Fisher's exact test when comparing proportions and two-factor ANOVA when comparing the means. CBCL scores showed a nonnormal distribution and to test for intervention group differences, Kruskal-Wallis rank-sum test was used. In cases of significant intervention group differences, unadjusted post hoc analyses were performed to explore intergroup differences. All analyses were performed on an intention to treat basis, meaning that the nine children who were prescribed to unblinded iron supplements at 12 weeks of age and the 43 cases from the 1- and 2-mg groups reporting poor compliance were included in the primary analyses. Twins (22\% of the subjects) were treated as independent participants in randomization and analyses.

In secondary analyses, all outcomes were also compared between the reference group and each intervention group, respectively, and the effect of intervention was explored stratifying for birth SGA/ appropriate for gestational age (AGA). Furthermore, as there were no observed differences between the 1- and 2-mg groups and to further explore the magnitude of the effect from iron supplements, we combined the two iron groups and compared their outcome to that 


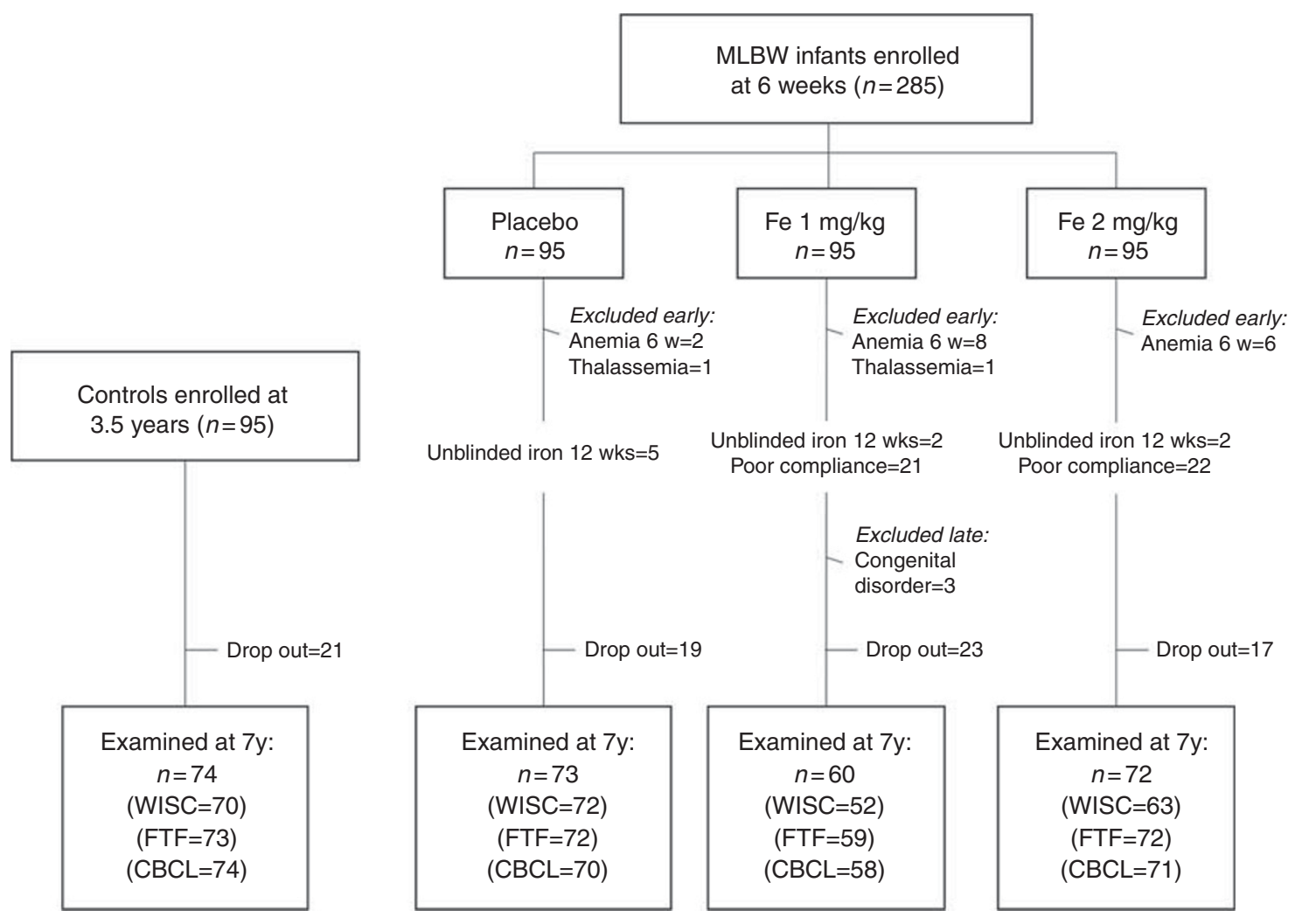

Figure 1. Trial profile.

of the placebo group. A logistic regression model was used to explore the correlation between early iron supplements and the risk of CBCL scores above each clinical cutoffs.

\section{RESULTS}

In total, $205(78 \%)$ of the 264 nonexcluded participants and $74(78 \%)$ children from the reference group were analyzed at the follow-up at 7 years of age (Figure 1). Baseline characteristics for these examined cases are presented in Table 1. There were no significant differences in any of the baseline characteristics between the 205 analyzed LBW children and the 59 dropouts or between the 74 analyzed references and the 21 dropouts. Of the analyzed LBW children, 95 were born as SGA and 95 were born as preterm, whereas 17 were both preterm and SGA.

At the 7-year follow-up, there were no significant differences in iron status between the intervention groups and their iron status was similar to the reference group (Supplementary Table S1 online). The results from the WISC-IV assessments are presented in Table 2. We found no significant differences between the intervention groups in any of the scales or between any of the intervention groups and the reference group. The results were similar in the subgroups born as SGA and AGA, respectively, with no significant interaction (data not shown).

The two parental scales of behavioral and emotional problems are presented in Table 3. Using CBCL, we observed a significant intervention group difference in
T-scores for externalizing behavior, with significantly higher scores in the placebo group compared with the two supplemented groups. The difference in T-scores for externalizing behavior was most pronounced in the LBW children born with AGA with a median (interquartile range) of $49(41 ; 55), 40.5(33 ; 49)$, and $41(34 ; 50)$ in the placebo, 1 $\mathrm{mg}$, and 2-mg group, respectively, where $P=0.10$. There was a similar trend in children born with SGA, and there was no significant interaction between AGA/SGA status and treatment when it came to externalizing behavior (data not shown).

The proportion of children with an internalizing, externalizing, and total behavioral problem score above the cutoff for clinical problems was the highest in the placebo group. However, when compared with the lower numbers observed in the iron-supplemented groups and in the reference group, none of the differences reached a statistical significance. In addition, in these dichotomized measures of behavior, the results were similar in the subgroups born with SGA and AGA, respectively (not shown).

With regard to FTF, there were no significant differences between the groups or between any of the intervention groups and the reference group. Furthermore, we did not observe any interaction with birth AGA/SGA.

When combining the 1- and 2-mg groups, comparing all iron-supplemented children with placebo, we found that the unsupplemented children had significantly higher CBCL scores for externalizing behavior (median (interquartile 


\section{Articles | Berglund et al.}

Table 1. Baseline characteristics of the marginally low-birth-weight children $(2,000-2,500 \mathrm{~g})$ and the reference group of normal-birth-weight children assessed at 7 years of age

\begin{tabular}{|c|c|c|c|c|c|}
\hline & Placebo; $n=73$ & $1 \mathrm{mg}$ of $\mathrm{Fe} / \mathrm{kg} / \mathrm{d} ; n=60$ & $2 \mathrm{mg}$ of $\mathrm{Fe} / \mathrm{kg} / \mathrm{d} ; n=72$ & $P$ & Reference; $n=74$ \\
\hline \multicolumn{6}{|l|}{ Sociodemographic background } \\
\hline Mother born in Scandinavia & $62(84.9 \%)$ & $52(86.7 \%)$ & $60(84.5 \%)$ & 0.939 & $68(93.2 \%)$ \\
\hline Maternal age at child birth (y) & $30.4(5.3)$ & $32.4(4.4)$ & $32.7(4.6)$ & 0.010 & $35.6(4.5)$ \\
\hline Mother educated at the university & $46(64.8 \%)$ & $36(61.0 \%)$ & 39 (55.7\%) & 0.556 & $43(58.9 \%)$ \\
\hline \multicolumn{6}{|l|}{ Perinatal characteristics } \\
\hline Vaginal delivery & $41(56.9 \%)$ & $35(58.3 \%)$ & $44(61.1 \%)$ & 0.905 & $52(71.2 \%)$ \\
\hline Single gestation & $50(68.5 \%)$ & $41(68.3 \%)$ & $53(73.6 \%)$ & 0.736 & $73(98.6 \%)$ \\
\hline Female sex & $38(52.1 \%)$ & $33(55.0 \%)$ & $33(45.8 \%)$ & 0.579 & 39 (52.7\%) \\
\hline Apgar score at $5 \mathrm{~min}$ & $9.5(1.0)$ & $9.5(1.0)$ & $9.5(1.1)$ & 0.922 & $9.8(0.5)$ \\
\hline Birth weight $(\mathrm{kg})$ & $2.28(0.15)$ & $2.27(0.16)$ & $2.31(0.14)$ & 0.230 & $3.55(0.43)$ \\
\hline Birth length $(\mathrm{cm})$ & $45.1(1.6)$ & $45.5(1.3)$ & $45.4(1.3)$ & 0.229 & $50.6(1.9)$ \\
\hline Head circumference at birth $(\mathrm{cm})$ & $32.1(1.2)$ & $32.2(1.0)$ & $32.2(1.3)$ & 0.856 & $35.1(1.5)$ \\
\hline Neonatal unit care & 31 (42.5\%) & $25(42.4 \%)$ & $41(56.9 \%)$ & 0.142 & $0(0 \%)$ \\
\hline Neonatal hypoglycemia & $11(15.1 \%)$ & $11(18.3 \%)$ & $15(20.9 \%)$ & 0.649 & $0(0 \%)$ \\
\hline Exclusively breastfed at 6 weeks & $41(56.9 \%)$ & $34(58.6 \%)$ & $39(54.2 \%)$ & 0.876 & Missing \\
\hline Exclusively breastfed at 6 months & $5(6.9 \%)$ & $2(4.2 \%)$ & $3(4.2 \%)$ & 0.716 & Missing \\
\hline
\end{tabular}

BMI, body mass index.

Mean (SD) or no. (\%).

$P$ value for differences between intervention groups (reference group not included) using Fisher's exact test for proportions, and ANOVA for means.

Table 2. Cognitive assessment using WISC-IV at 7 years in marginally low-birth-weight children supplemented with two doses of iron or placebo between 6 weeks and 6 months of age, and in same-aged controls with normal birth weight

\begin{tabular}{|c|c|c|c|c|c|}
\hline & Placebo; $n=71-72$ & $1 \mathrm{mg}$ of $\mathrm{Fe} / \mathrm{kg} / \mathrm{d} ; n=51-52$ & $2 \mathrm{mg}$ of $\mathrm{Fe} / \mathrm{kg} / \mathrm{d} ; n=62-63$ & $P$ & Reference; $n=69-70$ \\
\hline Verbal comprehension IQ & $104.7(8.8)$ & $104.9(9.3)$ & $103.7(10.0)$ & 0.746 & $107.2(10.2)$ \\
\hline Verbal comprehension IQ $<85$ & $1(1.4 \%)$ & $1(2.0 \%)$ & $2(3.2 \%)$ & 0.831 & $1(1.4 \%)$ \\
\hline Perceptual reasoning $\mathrm{IQ}<85$ & $2(2.8 \%)$ & $2(3.8 \%)$ & $3(4.8 \%)$ & 0.891 & $2(2.9 \%)$ \\
\hline Working memory IQ & $87.5(13.2)$ & $87.9(12.2)$ & $88.3(12.1)$ & 0.936 & $88.8(10.2)$ \\
\hline Processing speed $\mathrm{IQ}<85$ & $8(11.1 \%)$ & $6(11.5 \%)$ & $10(16.1 \%)$ & 0.676 & $9(12.9 \%)$ \\
\hline Full-scale IQ & $100.7(10.6)$ & $101.2(10.3)$ & $99.6(12.0)$ & 0.747 & $102.3(10.2)$ \\
\hline Full-scale $\mathrm{IQ}<85$ & $4(5.6 \%)$ & $1(2.0 \%)$ & $4(6.5 \%)$ & 0.577 & $3(4.3 \%)$ \\
\hline
\end{tabular}

IQ, intelligence quotient; WISC-IV, Wechsler Intelligence Scale for Children-fourth edition.

Mean (SD) or no. (\%).

$P$ value for differences between intervention groups (controls not included) using Fisher's exact test for proportions and ANOVA for means. No significant differences were observed between the normal-birth-weight reference group and each intervention group, respectively. 
Table 3. Behavioral and developmental assessment using the parental questionnaires CBCL and FTF at 7 years in marginally low-birth-weight children supplemented with different doses of iron between 6 weeks and 6 months of age

\begin{tabular}{|c|c|c|c|c|c|}
\hline & Placebo & $1 \mathrm{mg}$ of $\mathrm{Fe} / \mathrm{kg} / \mathrm{d}$ & $2 \mathrm{mg}$ of $\mathrm{Fe} / \mathrm{kg} / \mathrm{d}$ & $P$ & Reference \\
\hline$C B C L$ & $n=70$ & $n=58$ & $n=71$ & & $n=74$ \\
\hline Internalizing behavior, T-scores & $46(41 ; 50)$ & $45(39 ; 50)$ & $46(39 ; 52)$ & 0.789 & $43(37.7 ; 48.5)$ \\
\hline Internalizing problems, clinical & $7(10.0 \%)$ & $3(5.2 \%)$ & $4(5.6 \%)$ & 0.576 & $2(2.7 \%)$ \\
\hline Externalizing behavior, T-score & $48.5(41 ; 56)$ & $44(34 ; 51)^{a}$ & $44(40 ; 51)^{\mathrm{a}}$ & 0.045 & $44(34 ; 51)$ \\
\hline Total behavior, T-score & $45(38.7 ; 51)$ & $42.5(38 ; 49)$ & $43(38 ; 49)$ & 0.480 & $42(36 ; 47.5)$ \\
\hline Total behavioral problems, subclinical & $8(11.4 \%)$ & $5(8.6 \%)$ & $4(5.6 \%)$ & 0.460 & $2(2.7 \%)$ \\
\hline Total behavioral problems, clinical & $7(10.0 \%)$ & $1(1.7 \%)$ & $3(4.2 \%)$ & 0.125 & $2(2.7 \%)$ \\
\hline Executive functions & $0.32(0.41)$ & $0.30(0.37)$ & $0.25(0.32)$ & 0.479 & $0.26(0.34)$ \\
\hline Executive function impairment & $5(6.9 \%)$ & $3(5.1 \%)$ & $2(2.8 \%)$ & 0.717 & $4(5.5 \%)$ \\
\hline Perception & $0.20(0.21)$ & $0.19(0.18)$ & $0.18(0.18)$ & 0.801 & $0.19(0.22)$ \\
\hline Perception impairment & $1(1.4 \%)$ & $1(1.7 \%)$ & $2(2.8 \%)$ & 0.588 & $4(5.5 \%)$ \\
\hline Memory & $0.18(0.23)$ & $0.18(0.25)$ & $0.17(0.23)$ & 0.922 & $0.17(0.24)$ \\
\hline Memory impairment & $3(4.2 \%)$ & $1(1.7 \%)$ & $2(2.8 \%)$ & 0.898 & $2(2.7 \%)$ \\
\hline Language & $0.13(0.18)$ & $0.10(0.15)$ & $0.10(0.15)$ & 0.579 & $0.11(0.23)$ \\
\hline Language impairment & $4(5.6 \%)$ & $3(5.1 \%)$ & $3(4.2 \%)$ & 0.982 & $4(5.5 \%)$ \\
\hline Learning & $0.23(0.31)$ & $0.23(0.30)$ & $0.21(0.31)$ & 0.913 & $0.17(0.28)$ \\
\hline
\end{tabular}

CBCL, Child Behavior Checklist; FTF, Five to Fifteen.

Median (25th percentile; 75 th percentile), no. (\%), or mean (SD).

$P$ value for differences between intervention groups (controls not included) using Kruskal-Wallis rank-sum test for medians, Fisher's exact test for proportions, and ANOVA for means.

No significant differences were observed between the normal-birth-weight reference group and each intervention group, respectively.

a Significantly different from the placebo group in an unadjusted post hoc test.

range): 48.5 (41;56) vs. $44(34 ; 51), P=0.013)$. Furthermore, we found that the risk for a total behavioral score above the clinical cutoff in supplemented cases was reduced compared with those unsupplemented; however, this result was not statistically significant (relative risk 0.31 (95\% confidence interval: $0.09-1.02), P=0.054)$. When comparing the nine different subscales of $\mathrm{CBCL}$ between supplemented and nonsupplemented children, we not only observed a significant difference in the scores of aggressive and rule-breaking behavior, both included in the externalizing score, but also for thought problems (Figure 2).

Finally, we explored the association between the infant's iron status at 6 months of age and the present outcome at year 7. We found that none of the outcomes (WISC, CBCL, or FTF) were significantly different between those diagnosed with ID at 6 months $(n=31)$ vs. those diagnosed with non-ID $(n=165)$ or between those with a mean iron intake below $(n=104)$ or above $(n=71) 1 \mathrm{mg} / \mathrm{kg} /$ day $(18,23)$.

\section{DISCUSSION}

\section{Main Findings}

In this placebo-controlled trial, early iron supplementation did not affect the cognitive performance of 7-year-old children born as healthy marginally LBW infants. However, higher scores of adverse externalizing behavior (aggressive and rule-breaking behavior), as well as thought problems were found in the nonsupplemented children, in concordance with the effect that we previously reported in this cohort at 3.5 years (23). These findings suggest that, although early iron supplementation does not have an effect on cognition, it may 


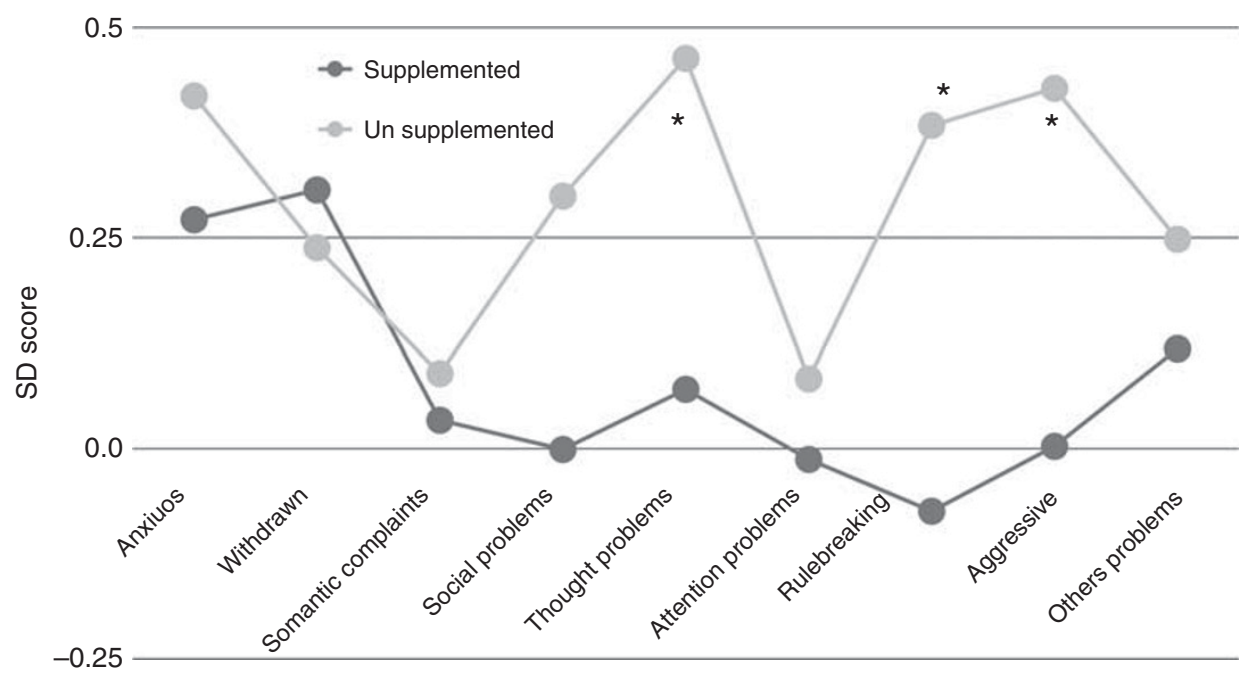

Figure 2. Child Behavior Checklist (CBCL) profile from the nine subscales in 7-year-old children randomized to placebo or iron supplementation (1 or $2 \mathrm{mg} / \mathrm{kg} /$ day) between 6 weeks and 6 months of age. SD scores were calculated based on a reference group of 74 normal-birth-weight children. Increased points represent increased behavioral problems. ${ }^{*} P<0.05$ (ANOVA).

reduce the long-term risk for behavioral problems in children born with marginally LBW to a level similar to that observed in children born with normal birth weight. Albeit not large, this effect might be clinically significant on the population level, as marginally LBW infants represent a relatively large proportion of all births $(19,20)$.

\section{Long-Term Outcome in Marginally LBW Infants}

Children born with marginally LBW are not a homogenous group. Both preterm and SGA infants fall into this definition. A substantial amount of research suggests poorer long-term development in late or moderately preterm infants compared with those born at term with normal birth weight. A difference averaging 2-3 IQ points has been reported in several studies (7-9). Similar developmental disadvantages have been shown in children born with SGA. According to a recent systematic review (38 studies included), children with intrauterine growth restriction (IUGR) had IQ scores that are on average $0.5 \mathrm{SD}$ lower than those without IUGR (0.7 SD if they were born at $<35$ weeks of gestation) (2). Here, we explored the hypothesis that inadequate availability of iron during the first months of life may contribute to this impairment. However, the results presented in this paper showed that iron supplementation during infancy did not change the IQ scores. This could be because of low power in the present study or, more likely, that iron supplements do not affect cognitive scores in this subgroup of LBW children. Moreover, the overall results of cognitive measures in children born with LBW were similar to the reference group. It is possible that the relatively high socioeconomic status and education level of participants' parents might have compensated for LBW as a risk factor for lower IQ. Comparison with other studies cannot be done, as this is the first iron supplementation trial with a neurodevelopmental follow-up in marginally LBW infants.
Problems related to behavior, attention, and emotions have been consistently shown to be more common in LBW and preterm children at school age (7-9). In Swedish children, preterm birth and early-term birth were independently associated with increased risk of ADHD by the degree of immaturity (3). A Dutch prospective twins study found that lower birth weight was a continuous risk factor for later child problem behavior. Moreover, the higher the birth-weight difference in a twin pair, the greater was the behavioral disadvantage in a smaller twin, suggesting lower weight at birth being an independent risk factor (5). In the US population, marginally LBW infants were $50 \%$ more likely to have attention deficit disorder/attention deficit hyperactivity disorder in later life than a normal-birth-weight reference group (4). We previously reported a reduced risk of behavioral problems at 3.5 years in marginally LBW infants who received iron supplementation from 6 weeks to 6 months of life (23). The results presented here suggest that this protective effect persists until 7 years of age, at least with regard to the externalizing behavior subscales. Even though the sample size limited the power to detect smaller differences, we observed significant differences in the CBCL scale for externalizing behavior, including aggressive and rule-breaking behavior. These externalizing subscales of CBCL have been strongly related with ADHD (28). However, the CBCL profile of the present cohort also showed increased scores of thought problems. This subscale was recently shown to be the best independent predictor of autism spectrum disorders, suggesting that the behavioral and emotional profile of non-iron-supplemented LBW children includes different symptoms of subclinical neurodevelopmental problems (29).

\section{The Role of Iron in the Developing Brain}

The substantial part of the brain tissue develops in the third trimester of pregnancy. The proposed risk-specific 
mechanisms of impairment in LBW infants are fetal malnourishment in those born with SGA, and aberrant brain development in premature infants (30). Gray and white matter injury has been associated to attention and executive function problems in preterm infants (31-33). Our results suggest that ID in infancy may also contribute to suboptimal brain development. The mechanistic support for this hypothesis is available from animal studies. Morphological brain alterations, impairment of myelination, and neurotransmitter system, along with the associated behavioral changes, were found in both rodent and mammal models of early ID (34). Less is known from human studies. Reduced iron concentrations in striatal and thalamic brain regions were displayed in magnetic resonance imaging images of children with ADHD compared with controls (35). A longterm effect of ID was suggested by a study in young adults, whose brain structure was associated with serum transferrin levels measured some years earlier (36).

Of note, in the present trial, iron status at 6 months was not correlated with behavioral outcome neither at 3.5 nor at 7 years. This may suggest that traditional measures of iron stores do not fully reflect the availability of iron for the developing brain. This is supported by recent animal studies that suggested that less iron is available for the brain tissue before ID can be detected in the blood by traditional measures: red blood cells production is then likely to be prioritized over brain supply (37).

\section{Strengths and Limitations}

The study has several strengths. First, it is a randomized controlled trial, which is the methodology of choice when studying the effects of treatment or prevention. Randomization ensures that any differences with regard to known or unknown confounding factors that may occur between study groups (e.g., sociodemographic factors) are due to chance, so that the risk that they would affect the outcome is limited. To our knowledge, this is the first randomized trial investigating the effect of iron supplementation on neurodevelopmental outcome in LBW infants until school age. Follow-up of 78\% of the participants 7 years after inclusion and compliance to the study protocol in $76 \%$ of the participants are additional methodological strengths. Both dropout rates and the number of noncompliant participants did not differ between the trial arms. Second, the study group represents a relatively large part of the general population, including both late preterm and term SGA infants. The lack of significant interactions with birth SGA suggests that the results observed here can be applied on the whole to this large and clinically wellrecognized population. Finally, the normal-birth-weight reference group was recruited from the same population providing a well-matched reference.

The study also has some limitations. It was underpowered to detect differences below $0.5 \mathrm{SD}$, a problem that was partly reduced by combining the two iron-supplemented groups in the secondary statistical analyses. Behavioral outcome was based on questionnaires filled in by parents that limits the specificity and sensitivity for these assessments. However, the questionnaires have been validated and used in several previous studies. Allocation concealment and blinding of caregivers further limited the risk for biased results. Another limitation of our study is that it was designed to study the effect of intervention in this group as a whole, and the relatively low sample size limited the possibilities for further stratified analyses. However, as no adverse effects were observed, we find it reasonable to apply the results to all otherwise healthy or marginally low-birth-weight infants.

\section{CONCLUSIONS}

The present study showed that early iron supplementation in marginally LBW infants may prevent behavioral problems at school age. In addition to prevention of ID anemia, this effect should be acknowledged as a clinically important benefit from early iron supplementation, and it gives further support to recommend iron supplementation of all LBW children. Further research should focus on strategies to optimize the cognitive performance and behavior in children with marginally LBW, including the assessment of the optimal dose and duration of iron supplementation.

\section{SUPPLEMENTARY MATERIAL}

Supplementary material is linked to the online version of the paper at http://www.nature.com/pr

\section{ACKNOWLEDGMENTS}

We want to thank all participating children and their families, and acknowledge our dedicated research nurses Kerstin Andersson in Stockholm and Åsa Sundström in Umeå.

\section{STATEMENT OF FINANCIAL SUPPORT}

This work was supported by grants from the Swedish Research Council (Formas-222-2005-1894), Swedish Research Council for Health, Working Life, and Welfare (FORTE-2012-0708), the Jerring Foundation, the Oskar Foundation, and The Swedish Society of Medicine (SLS-331751). The Childhood Foundation of the Swedish Order of Freemasons, by a regional agreement between Umeå University and Västerbotten County Council (ALF) and by a regional agreement on clinical research (ALF) between Stockholm County Council and Karolinska Institutet.

\section{DISCLAIMER}

The funders had no role in the study design, data collection and analysis, decision to publish, or preparation of the manuscript.

Disclosure: The authors declare no conflict of interest.

\section{REFERENCES}

1. Bhutta AT, Cleves MA, Casey PH, Cradock MM, Anand KJ. Cognitive and behavioral outcomes of school-aged children who were born preterm: a meta-analysis. JAMA 2002;288:728-37.

2. Murray E, Fernandes M, Fazel M, Kennedy SH, Villar J, Stein A. Differential effect of intrauterine growth restriction on childhood neurodevelopment: a systematic review. BJOG 2015;122:1062-72.

3. Lindstrom K, Lindblad F, Hjern A. Preterm birth and attention-deficit/ hyperactivity disorder in schoolchildren. Pediatrics 2011;127:858-65.

4. Boulet SL, Schieve LA, Boyle CA. Birth weight and health and developmental outcomes in US children, 1997-2005. Matern Child Health J 2009;15:836-44. 


\section{Articles | Berglund et al.}

5. van Os J, Wichers M, Danckaerts M, Van Gestel S, Derom C, Vlietinck R. A prospective twin study of birth weight discordance and child problem behavior. Biol Psychiatry 2001;50:593-9.

6. Gray RF, Indurkhya A, McCormick MC. Prevalence, stability, and predictors of clinically significant behavior problems in low birth weight children at 3, 5, and 8 years of age. Pediatrics 2004;114:736-43.

7. van Baar AL, Vermaas J, Knots E, de Kleine MJ, Soons P. Functioning at school age of moderately preterm children born at 32 to 36 weeks' gestational age. Pediatrics 2009;124:251-7.

8. Talge NM, Holzman C, Wang J, Lucia V, Gardiner J, Breslau N. Latepreterm birth and its association with cognitive and socioemotional outcomes at 6 years of age. Pediatrics 2010;126:1124-31.

9. Cserjesi R, Van Braeckel KN, Butcher PR, et al. Functioning of 7-year-old children born at 32 to 35 weeks' gestational age. Pediatrics 2012;130: e838-46.

10. Berglund S, Domellof M. Meeting iron needs for infants and children. Curr Opin Clin Nutr Metab Care 2014;17:267-72.

11. Domellof M, Braegger C, Campoy C, et al. Iron requirements of infants and toddlers. J Pediatr Gastroenterol Nutr 2014;58:119-29.

12. Lozoff B, Beard J, Connor J, Barbara F, Georgieff M, Schallert T. Longlasting neural and behavioral effects of iron deficiency in infancy. Nutr Rev 2006;64:S34-43; discussion S72-S91.

13. Baker RD, Greer FR. Diagnosis and prevention of iron deficiency and iron-deficiency anemia in infants and young children (0-3 years of age). Pediatrics 2010;126:1040-50.

14. Sazawal S, Black RE, Ramsan M, et al. Effects of routine prophylactic supplementation with iron and folic acid on admission to hospital and mortality in preschool children in a high malaria transmission setting: community-based, randomised, placebo-controlled trial. Lancet 2006;367: 133-43.

15. Dewey KG, Domellof M, Cohen RJ, Landa Rivera L, Hernell O, Lonnerdal B. Iron supplementation affects growth and morbidity of breast-fed infants: results of a randomized trial in Sweden and Honduras. J Nutr 2002;132:3249-55.

16. Lozoff B, Castillo M, Clark KM, Smith JB. Iron-fortified vs low-iron infant formula: developmental outcome at 10 years. Arch Pediatr Adolesc Med 2012;166:208-15.

17. Lozoff B, Jiang Y, Li X, et al. Low-dose iron supplementation in infancy modestly increases infant iron status at 9 mo without decreasing growth or increasing illness in a randomized clinical trial in rural China. J Nutr 2016;146:612-21.

18. Berglund S, Westrup B, Domellof M. Iron supplements reduce the risk of iron deficiency anemia in marginally low birth weight infants. Pediatrics 2010;126:e874-83.

19. Office for National Statistics Birth Statistics: Review of the National Statistician on Births and Patterns of Family Building in England and Wales, 2007. Newport: Officefor National Statistics, 2008. Series FM1 No. 36 (https://www.gov.uk/government/statistics/birth-statistics-review-ofthe-national-statistician-on-births-2007). Accessed 4 May 2017..

20. Hamilton BE, Martin JA, Osterman MJ, Curtin SC, Matthews TJ. Births: final data for 2014. Natl Vital Stat Rep 2015;64:1-64.
21. United Nations Children's Fund (UNICEF) The State of the World's Children 2009: Maternal and Newborn Health. New York: United Nations Children's Fund. 2008 (http://www.unicef.org/sowc09/docs/ SOWC09-FullReport-EN.pdf). Accessed 4 May 20017.

22. Berglund SK, Westrup B, Domellof M. Iron supplementation until 6 months protects marginally low-birth-weight infants from iron deficiency during their first year of life. J Pediatr Gastroenterol Nutr 2015;60:390-5.

23. Berglund SK, Westrup B, Hagglof B, Hernell O, Domellof M. Effects of iron supplementation of LBW infants on cognition and behavior at 3 years. Pediatrics 2013;131:47-55.

24. D W Weschsler Intelligence Scale for Children-Fourth Edition (WISCIV). Antonio, TX: The Psychological Corporation, 2003.

25. Achenbach TM, Rescorla LA. (eds). Manual for the ASEBA School-Age Forms \& Profiles. Burlington, VT: University of Vermont, Research Center for Children, Youth, \& Families, 2001.

26. Kadesjo B, Janols LO, Korkman M, et al. The FTF (Five to Fifteen): the development of a parent questionnaire for the assessment of ADHD and comorbid conditions. Eur Child Adolesc Psychiatry 2004;13 (Suppl 3): 3-13.

27. Achenbach TM, Becker A, Dopfner M, et al. Multicultural assessment of child and adolescent psychopathology with ASEBA and SDQ instruments: research findings, applications, and future directions. J Child Psychol Psychiatry 2008;49:251-75.

28. Kuja-Halkola R, Lichtenstein P, D'Onofrio BM, Larsson H. Codevelopment of ADHD and externalizing behavior from childhood to adulthood. J Child Psychol Psychiatry 2015;56:640-7.

29. Biederman J, Petty CR, Fried R, et al. Child behavior checklist clinical scales discriminate referred youth with autism spectrum disorder: a preliminary study. J Dev Behav Pediatr 2010;31:485-90.

30. Inder TE, Warfield SK, Wang H, Huppi PS, Volpe JJ. Abnormal cerebral structure is present at term in premature infants. Pediatrics 2005;115: 286-94.

31. Nosarti C, Giouroukou E, Healy E, et al. Grey and white matter distribution in very preterm adolescents mediates neurodevelopmental outcome. Brain 2008;131:205-17.

32. Kinney HC. The near-term (late preterm) human brain and risk for periventricular leukomalacia: a review. Semin Perinatol 2006;30:81-8.

33. Brumbaugh JE, Conrad AL, Lee JK, et al. Altered brain function, structure, and developmental trajectory in children born late preterm. Pediatr Res 2016;80:197-203.

34. Lozoff B. Early iron deficiency has brain and behavior effects consistent with dopaminergic dysfunction. J Nutr 2011;141:740S-6SS.

35. Adisetiyo V, Jensen JH, Tabesh A, et al. Multimodal MR imaging of brain iron in attention deficit hyperactivity disorder: a noninvasive biomarker that responds to psychostimulant treatment? Radiology 2014;272:524-32.

36. Jahanshada N, Kohannim O, Hibara DP, et al. Brain structure in healthy adults is related to serum transferrin and the H63D polymorphism in the HFE gene. Proc Natl Acad Sci USA 2012;109:E851-9.

37. Zamora TG, Guiang SF 3rd, Widness JA, Georgieff MK. Iron is prioritized to red blood cells over the brain in phlebotomized anemic newborn lambs. Pediatr Res 2016;79:922-8. 